\title{
Inelastic neutron scattering as a confirmation of a new type of gapped surface excitations in liquid helium
}

\author{
P. D. Grigoriev ${ }^{1,2}$, A. D. Grigoriev ${ }^{3}$, A. M. Dyugaev ${ }^{1}$ \\ ${ }^{1}$ L.D. Landau Institute for Theoretical Physics, Chernogolovka, Russia \\ ${ }^{2}$ National University of Science and Technology "MISiS", 119049 Moscow, Russia and \\ ${ }^{3}$ Samara State Technical University, 443100, Samara, Russia
}

(Dated: November 13, 2018)

\begin{abstract}
We analyze the experimental data [1] on inelastic neutron scattering by a thin 5-atomic-layer film of liquid helium at three different temperatures: $\mathrm{T}=0.4 \mathrm{~K}, 0.98 \mathrm{~K}$ and $1.3 \mathrm{~K}$. These data were partially published previously, 2- 4] but here we present them in a better quality and at various temperatures. The neutron scattering intensity plots, in addition to the previously know dispersion of phonons and ripplons, suggest a branch of gapped surface excitations with activation energy $\sim 4.5 \mathrm{~K}$ and the dispersion similar to that expected for surfons - the bound quantum states of helium atoms above liquid helium surface, proposed and investigated theoretically [5, [6]. These data, probably, provide the first direct experimental confirmation of surfons. Before these surface excitations received only indirect experimental substantiation, based on the temperature dependence of surface tension coefficient [5, 6] and on their interaction with surface electrons [7, 8]. The existence of surfons as an additional type of surface excitations, although being debated yet, is very important for various physical properties of He surface. We also analyze previous numerical results on excitations in liquid helium and argue that surface excitations similar to surfons have been previously obtained by numerical calculations and called resonance interface states [21].
\end{abstract}

\section{INTRODUCTION}

A deep understanding of the processes on the surface of liquids is important for various fields of natural science: physics, chemistry, biology. The microscopic description of liquid surface is a rather complicated problem, and various theoretical techniques have been applied to advance it. 9] At low temperature the quantum nature of surface excitations is important, which becomes apparent in liquid helium and can be experimentally studied, e.g., using the interaction of these excitations with surface electrons [10 13]. In bulk liquid helium the excitations are well known both from microscopic theory [14, 15] and from the extensive thermodynamic and neutron scattering experiments. The microscopic description of the surface excitations in liquid helium is more complicated because of spatial inhomogeneity of this problem. This problem was quite successfully studied using the numerical variation methods with the Feenberg wave function in the so-called hypernetted-chain approximation [16-21]. These numerical results were used to analyze the experimental data on inelastic neutron scattering by liquid helium films, [24, 22, 23. the temperature dependence of the surface tension coefficient [24] and other thermodynamic properties [25].

In liquid ${ }^{4} \mathrm{He}$ there is only one type of gapless surface excitations - the quanta of surface waves, called ripplons. For the wavelength much larger than interatomic distance but smaller than the capillary length $\kappa^{-1} \approx 0.05 \mathrm{~cm}$, the dispersion of surface waves is given by 26 ]

$$
\omega_{q}^{2}=\frac{\alpha}{\rho} q^{3} \tanh (q d)
$$

where $\alpha$ is surface tension coefficient, $\rho$ is the density of liquid, $q$ is the ripplon wave number, and $d$ is the width of liquid helium film. For short-wave-length ripplons with $q \gtrsim 1 \AA^{-1}$ the ripplon dispersion $\omega(q)$ becomes softer than in Eq. (1) and saturates at energy $\hbar \omega_{D} \approx 0.8 \mathrm{meV} \approx 10 \mathrm{~K}$, which was obtained numerically [16 21] and observed using the inelastic neutron scattering by liquid helium films. [2-4]

Recently, an additional new type of surface excitations has been proposed semi-phenomenologically to explain too strong temperature dependence of surface tension coefficient $\alpha(T) .[5,6]$ These excitations, called surfons, can be considered as the quantum states of He atoms localized above liquid surface. Surfons resemble the Andreev states of ${ }^{3} \mathrm{He}$ atoms in the ${ }^{3} \mathrm{He}-{ }^{4} \mathrm{He}$ mixture [27], or the states of ${ }^{3} \mathrm{He}$ and ${ }^{4} \mathrm{He}$ atoms on the surface of liquid hydrogen. 28 According to this phenomenological model [5, 6], the surfons are localized only along the $z$-axis perpendicular to helium surface and can propagate along the surface. Their dispersion is

$$
\varepsilon(k) \approx \Delta+k_{\|}^{2} / 2 M^{*},
$$

where $k_{\|}$is a 2D surfon momentum along the surface, $\Delta>0$ is the activation energy of a surfon at $k_{\|}=0$, and their effective mass $M^{*}$ is of the order of the atomic $\mathrm{He}$ mass $M_{4}^{0}=6.7 \cdot 10^{-24} \mathrm{~g}$. The surfon activation energy $\Delta$ is weakly temperature-dependent,

$$
\Delta(T)=E_{s}-\mu(T)
$$

where $\mu(T)$ is the temperature-dependent chemical potential of a liquid ${ }^{4} \mathrm{He}, \mu(T=0) \approx-7.17 K$, and $E_{s}$ is the discrete energy level of $\mathrm{He}$ atom at liquid surface, dressed by the interaction with other atoms, ripplons and phonons. At low temperature $T \ll \Delta$ the surfon concentration is exponentially small. 
Currently there are several experimental facts which can be treated as indirect substantiation of surfons. The first two are related to the interaction of surface electrons with surfons, which provides an additional temperaturedependent scattering mechanism of surface electrons. This additional scattering mechanism can considerably improve [7] the agreement between the observed [29] and calculated [30] mobility of surface electrons. Surfons can also explain the observed [11] temperature-dependent shift of the transition line between two lowest electron states above liquid helium or solid hydrogen surface. [8] Finally, the surfons can explain [5, 6] the long-standing puzzle of the observed [31] too strong temperature dependence $\alpha(T)$ of the surface tension coefficient of liquid He. The comparison can be used to estimate the surfon activation energy for both He isotopes from experiment as [6]

$$
\Delta^{H e 4} \approx 2.67 K, \Delta^{H e 3} \approx 0.25 K
$$

corresponding to $E_{s}^{H e 4} \approx-4.5 K$ and $E_{s}^{H e 3} \approx-2.25 K$. This value $\Delta^{H e 4} \approx 2.67 K$, obtained from the fitting of $\alpha(T),[\overrightarrow{6}]$ is in a reasonable agreement with the energy gap $\Delta_{2} \approx 1.6 \mathrm{~K}$ of resonance interface states obtained from the numerical calculations in Ref. [21] (see below) and with $\Delta^{H e 4} \approx 3.2 \mathrm{~K}$ obtained from the semiphenomenological description in Ref. [6]. Fitting the surface tension of a thick He film, assuming that only ripplons and surfons make considerable contribution, gives an upper estimate of the surfon effective mass: [6] $M_{4}^{*} \approx$ $2.65 M_{4}^{0}$, and $M_{3}^{*} \approx 2.25 M_{3}^{0}$, where $M_{4}^{0}=6.7 \cdot 10^{-24} \mathrm{~g}$ and $M_{3}^{0}=5.05 \cdot 10^{-24} \mathrm{~g}$ are the free atomic masses of ${ }^{4} \mathrm{He}$ and ${ }^{3} \mathrm{He}$ correspondingly. The effective surfon mass $M^{*}$ in its in-plane motion is, probably, renormalized by interaction with liquid. Note, that the inclusion of the second branch of surface excitations, obtained numerically in Ref. [20], also considerably improves the agreement between experiment [31] and theory [24] on $\alpha(T)$ of thick He films. The temperature dependence of surface tension coefficient $\alpha(T)$ of thin He films cannot also be fitted without additional type of surface excitations, though the "breathing modes" (or "quantized bulk phonons"), obtained in Refs. 16 18, 22] make a considerable contribution [33] to $\alpha(T)$.

The microscopic substantiation of the surfon existence was provided [6] by the solution of one-particle Schrödinger equation for a He atom above liquid He surface in the effective one-dimensional potential $V(z)$ created by the interaction with other He atoms in the lower half-space (Hartree approximation). The corresponding one-particle Schrödinger equation is uniform in the $x$ $y$ plane and does not take into account the correlation effects. However, it definitively gives a discrete quasistationary energy level $E_{s} \approx-1.24 K<0$ of a He atom above the surface. The neglected correlation effects in liquid $\mathrm{He}$, which partially may be treated as a "dressing" of a surfon by ripplonic polaron, reduce considerably the value $E_{s}$ of this quasi-stationary energy level to $E_{s} \approx-4 K$, as was semi-phenomenologically estimated in
Ref. [6], but they do not destroy these excitations. This quasi-stationary level also persists after the inclusion of exchange interaction between $\mathrm{He}$ atoms. The latter is weak for liquid ${ }^{4} \mathrm{He}$ because the wave functions of $\mathrm{He}$ atoms overlap weakly due to their strong hard-core repulsion at distance $z<2.5 \AA$.

The surfon lifetime $\tau$ is rather short and limited mainly by two processes: the immersion into liquid and evaporation due to scattering by other excitations. The second process was studied in Ref. [32]. The evaporation rate $1 / \tau_{v}$ of surfons depends on their initial momentum along the surface and grows rapidly with the increase of temperature. 32. However, below $4 \mathrm{~K}$ it does not exceed the limit $\sim E_{s} / \hbar$ where the surfons cannot be called quasiparticles. The immersion rate of surfons to the liquid has not been calculated yet, but it should also be less than $E_{s} / \hbar$, because in order to sink into the liquid, a $\mathrm{He}$ atom must overcome a potential barrier and rearrange the surrounding atoms of the liquid.

Thus, the surfons are, presumably, non-stable quasiparticle with lifetime shorter than that of long-wavelength ripplons or phonons. Nevertheless, the existence of surfons as an additional type of surface excitations, although being debated yet, is crucial for various physical properties of He surface. In addition to explaining the strong temperature dependence of the surface tension coefficient, [5, [6] they may considerably increase the evaporation rate of liquid He by adding a new evaporation channel via the intermediate surfon state with activation energy $\Delta$ smaller than the evaporation energy $|\mu|$.[32] The surfon quasi-stationary quantum states may also affect the reflection coefficient of He atoms by liquid He surface [34]. Therefore, any substantiation of this new type of excitations, experimental or theoretical, is very important. At the moment there are only indirect experimental confirmations of the existence of surfons by their interaction with surface electrons $[7,8$ or their contribution to surface tension coefficient [ $[5,6]$. In this paper we analyze the experimental data on inelastic neutron scattering by thin He films and study if these data can provide a direct experimental confirmation of the existence of surfons. We also summarize the available results of ab-initio numerical calculations of surface excitations in liquid $\mathrm{He}$, which also indicate the existence of surfons.

\section{EXPERIMENTAL DATA AND THEIR ANALYSIS}

In this section we present and analyze the experimental data, obtained by the group of H. Godfrin [1] and shown in Figs. 1, 3] on inelastic neutron scattering by a thin 5-atomic-layer film of liquid helium at three different temperatures: $\mathrm{T}=0.4 \mathrm{~K}, 0.98 \mathrm{~K}$ and $1.3 \mathrm{~K}$. Similar and even these data were partially published previously in Refs. [2 4] , but here we present them at different temperatures, in a better quality and in color for greater visibility and resolution [1]. 


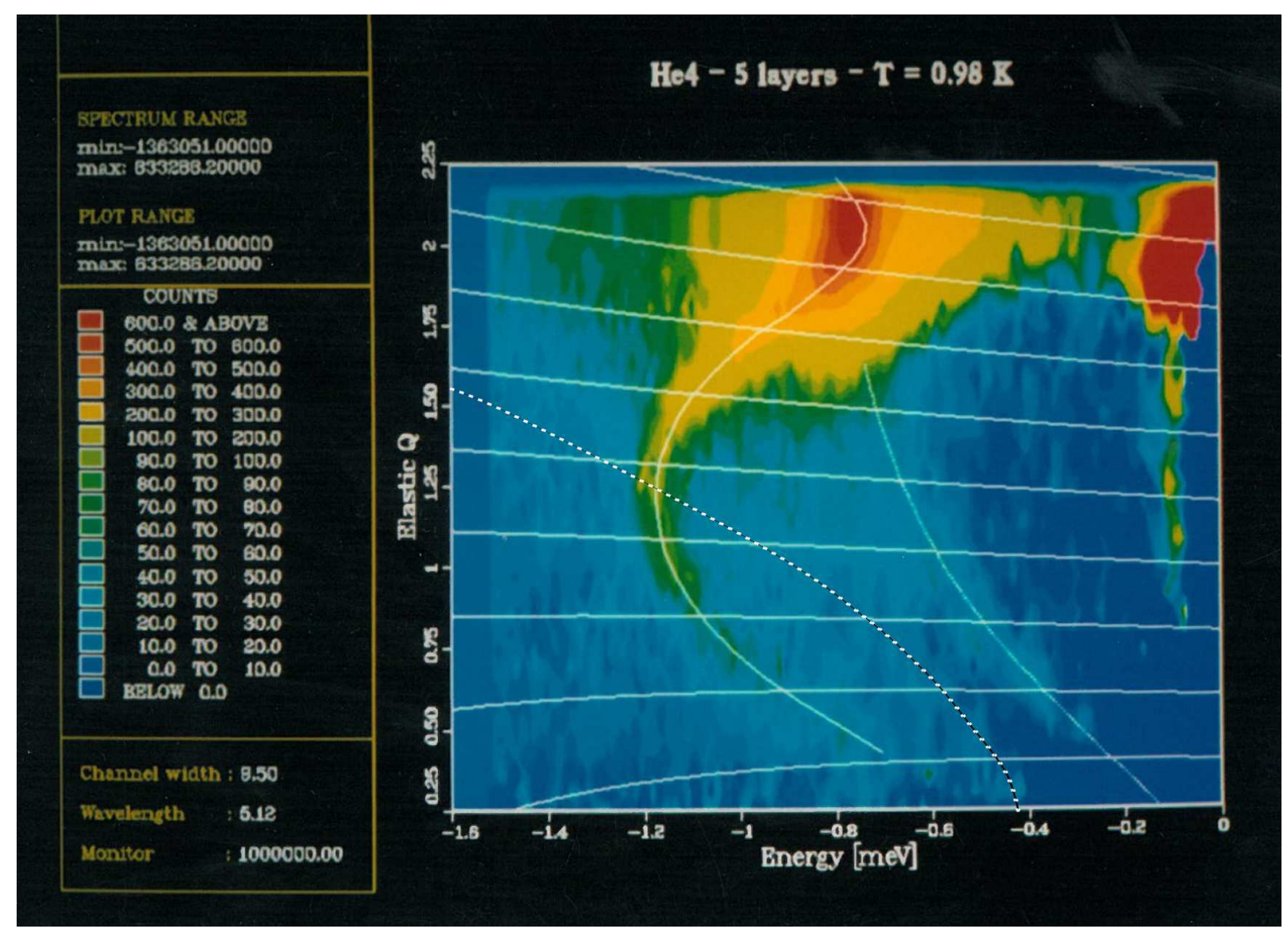

FIG. 1: (Color on-line) Experimental data on neutron inelastic scattering intensity by liquid helium film, containing only 5 atomic layers at $T=0.98 \mathrm{~K}$, as a function of the in-plane momentum $Q=q_{\|}$and of the energy transfer. The solid white lines along the intensity maxima mark the phonon and ripplon spectra. The white dashed line corresponds to the expected dispersion of surfons.

The experimental setup and method were described previously in detail. 22-4] Helium was adsorbed onto a substrate of exfoliated graphite. The He film of the thickness of approximately 5 atomic layers has been used, because for thicker films the contribution of surface excitations is too weak as compared to the dominant contribution from the bulk excitations (phonons). Thinner films also have drawbacks for the study of surfons. First, the 2-3 atomic layers adjacent to the substrate are solid and their structure differs considerably from that in the bulk He. Second, due to the dimensional quantization along z-axis, the bulk excitations in too thin films may also contain the energy gap $\sim 1 K$ and resemple the surfons. The inelastic neutron-scattering experiments were performed at the ILL on the time-of-flight spectrometer IN6 with an incident wavelength of $5.12 \AA$. The detectors were located in an angular range, corresponding to momentum transfers between $0.254 \AA^{-1}$ and $2.046 \AA^{-1}$ for elastically scattered neutrons. The energy resolution was $\sim 0.6-0.7 \mathrm{~K}$ and depended only slightly on the momentum transfer.
In Ref. [2-4] the authors studied mainly the ripplon and phonon spectra in several He films of various thickness and in Ref. [4] in the shorter interval $q_{\|}>0.4 \AA^{-1}$ of in-plane wave-vector. In this paper we present these experimental data in a full available wave-vector interval $q_{||}>0.25 \AA^{-1}$ and concentrate on the momentum-energy region corresponding to the expected dispersion of surfons. In addition, we provide partially unpublished data [1] at three different temperatures of liquid He, namely, $T=0.4 K, 0.98 K$ and $1.3 K$, while in Refs. 2-4] only the data at $T=0.65 K$ are given.

The intensity of neutron scattering in Figs. 1-3 as a function of the energy $\hbar \omega$ and in-plane wave-vector $q_{\|}$ of induced excitations is given by color (brightness in greyscale) as shown on the left panel of Fig. 1. The bright areas form thick lines in the $q_{\|}-\omega$ coordinates, which give the dispersion relation of excitations. In all Figs. 1.3. one can easily distinguish the ripplon branch (lowest curve) and the phonon branch (upper curve), marked by white thin curves. The phonon and ripplon modes agree 


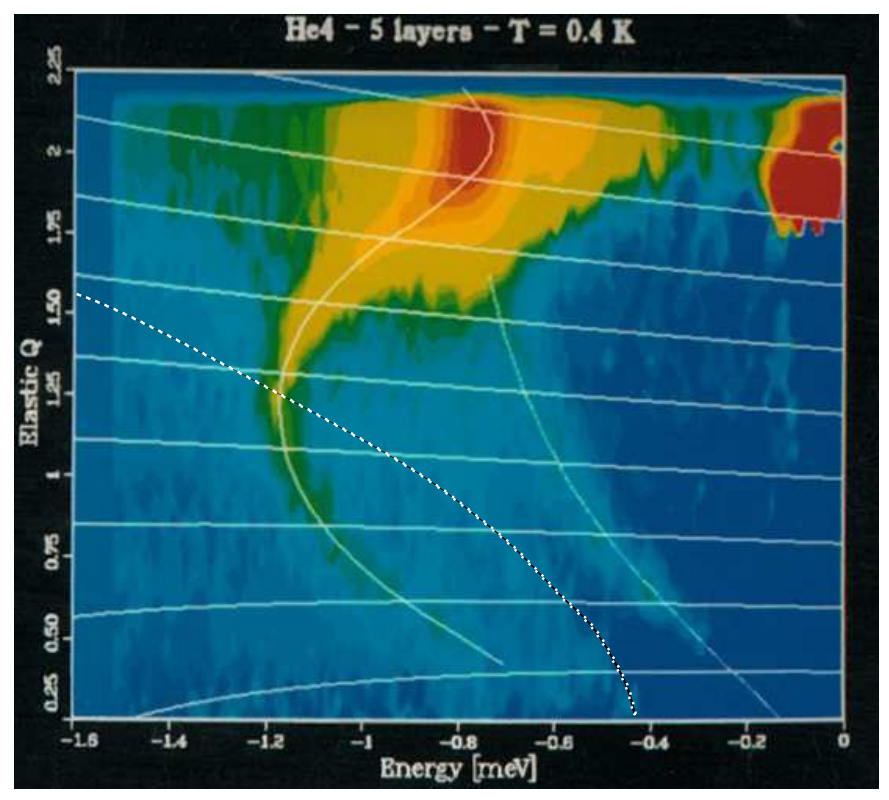

FIG. 2: (Color on-line) Experimental data on neutron inelastic scattering intensity by liquid helium film, containing only 5 atomic layers at $T=0.4 K$, as function of the in-plane momentum $Q=q_{\|}$and of energy transfer. The white dashed line corresponds to the expected dispersion of surfons.

with those in Refs. 2-4]. The phonon branch has a roton minimum at $q_{\|} \approx 2 \AA^{-1}$, which gives a strong intensity maximum of inelastic neutron scattering. In addition to these two well-know excitation branches, on each of Figs. 1 13 one can distinguish another curve of intensity maxima, located at $0.25 \AA^{-1}<q_{\|}<1.5 \AA^{-1}$ between the phonon and ripplon branches and approximately coinciding with the white dashed line of expected surfon dispersion. The intensity of neutron scattering (brightness) of this curve is beyond the error-bar [1] and at small wave-vector is even stronger than that of ripplons on all three Figs. 1]3, This intermediate dispersion curve gives a gapped excitation and, possibly, originates from surfons, because the white dashed lines in Figs. 1] 3 show the expected surfon dispersion given by Eq. (2) with the activation energy $\Delta \approx 4.5 \mathrm{~K}$. The corresponding effective mass of surfon branch coincides with the mass of a free He atom, $M^{*}=M_{4}^{0}$, because the inelastic neutron scattering is a process of short time $\sim \hbar / \varepsilon<\hbar / \Delta$, while the "dressing" of surfons by the formation of the ripplonic polaron (dimple), leading to the increase of surfon effective mass, [6] requires longer time. Note that some traces of this additional branch of surface excitations are seen already in Fig. 1 of Ref. [2], but in the Figs. 1] 3 of current paper this branch is clearer. Thus, the available experimental data on inelastic neutron scattering by thin He films support the existence of surfons [5-8] as an additional type of surface excitations.

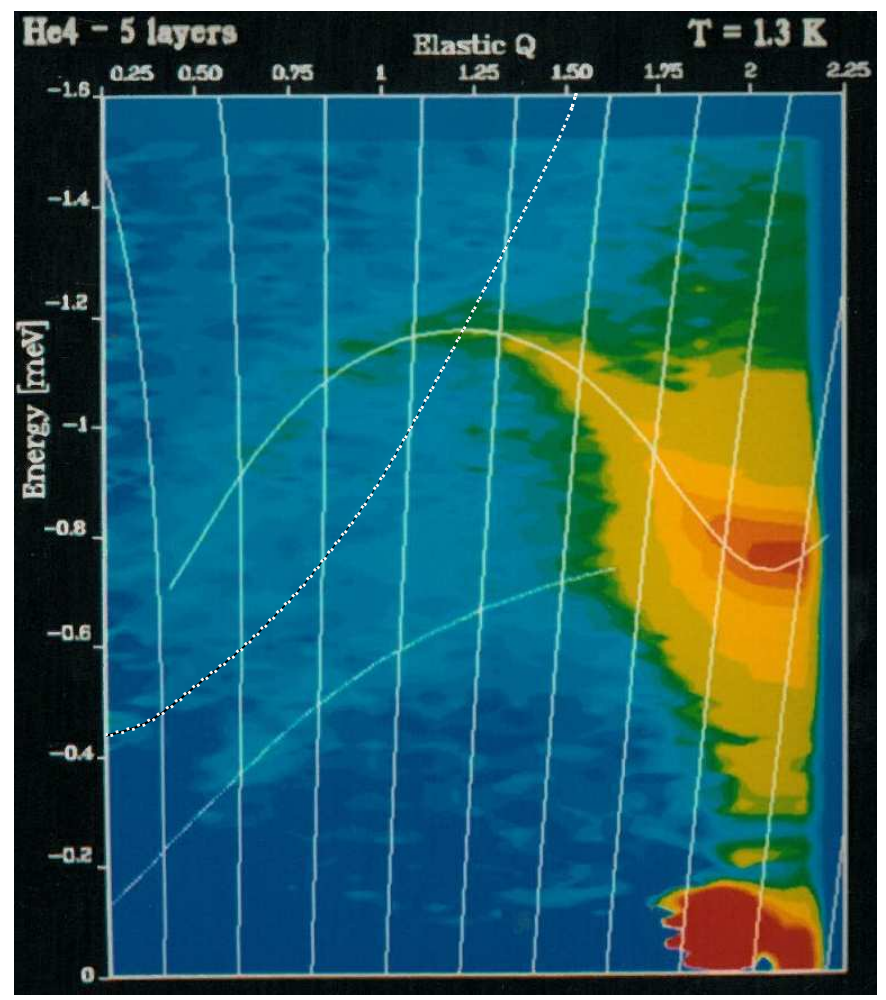

FIG. 3: (Color on-line) The same as in Figs. 1,2 but at different temperature $T=1.3 \mathrm{~K}$ and rotated by $90^{\circ}$.

\section{COMPARISON OF VARIOUS THEORETICAL CALCULATIONS OF SURFACE EXCITATIONS IN HELIUM AND DISCUSSION}

The microscopic numerical calculations also propose several types of surface excitations in addition to ripplons. 16-22] These numerical calculations apply the correlated basis function (CBF) method [15] for inhomogeneous liquid, using some additional approximations. These CBF calculations are based on the Feenberg wave function with only pair correlations and performed in the hypernetted-chain approximation. These calculations also assume that only one-body component of the Feenberg function is affected by excitations and by external perturbations. 16. This assumption of static twobody correlations limits the regime of validity of this theory to wavelengths longer than the average distance between two particles. It also may restrict the theory to small deviations from the equilibrium (ground-state) density of liquid He. The backflow effects [14] are also ignored in these numerical calculations. Therefore, the obtained theoretical excitation energies calculated at large wave numbers $q_{\|}$are substantially higher than the experimental results. [20] Since the applied Jastrow variational treatment of the bulk liquid does not produce a self-bound system at saturation He density, an external potential is introduced phenomenologically in these numerical calculations to stabilize the surface. [20, 21] 
The strength of this additional phenomenological potential is adjusted so that the calculated chemical potential matches the experimental saturation value.[20] Finally, the standard numerical computations assume that surface excitations do not violate the translational symmetry along the surface, which may not describe the case of a single surfon with zero in-plane momentum. Nevertheless, the comparison of the surface excitations proposed by these approximate numerical calculations with the surfons proposed semi-phenomenologically [5, 6] is quite useful.

In Refs. 16 18, 22 the ground state and excitations in thin He film of several atomic layers on a substrate of various materials were investigated, and several types of excitations in He films with a non-zero gap were found. The analysis of the particle currents and transition densities, in addition to the dispersion relation of these excitations, allowed to describe their nature: 22] they were attributed to the ripplon mode on the He-substrate interface and to the so-called "breathing mode". The latter describes the standing wave in the $z$-direction perpendicular to the film, 22] similar to the volume phonon, which may propagate along the film. The energy gap of this "breathing mode" reduces with the increase of the film thickness. Thus, no one of the excitations found in Refs. [16 18, 22] can be attributed to surfons. The reason is that these calculations [16 18, 22] include only excitations inside the liquid, neglecting He vapor and the states above liquid He. This restriction was eliminated in Refs. 20, 21], where a free boundary between a deep liquid He and saturated vapor was investigated by the similar numerical microscopic CBF approach, and notably different results were obtained. This limit of deep liquid He, filling a half-space instead of atomically thin film, is closer to the model of semi-phenomenological description of surfons in Ref. [6]. Again several gapped surface excitations were obtained in deep liquid He [20, 21], but the structure of these excitations is completely different from those in thin films [16 18, 22]. First, no signature of "breathing mode" was found at the free surface of bulk $\mathrm{He},[20,21]$ which is natural because this mode was found to spread along the whole He film thickness 22], being rather the bulk excitation. Nevertheless, two new types of gapped surface excitations in deep He were found. [21] The first type has a large excitation energy above $\Delta_{1} \approx 18.5 K$ and is interpreted as a bound roton trapped in the interface region. 21] The wave function of this excitation is mostly inside liquid He (see Fig. 2 of Ref. [21]), so it cannot be interpreted as the surfon.

The second type of gapped surface excitations, found in Ref. 21] and called resonance interface state (RIS), has the structure and properties very similar to those of surfons. First, RIS correspond to a peak of He density just above the liquid surface, as shown in Fig. 6 of Ref. 21]. This is very similar to the wave function of surfons, shown in Fig. 1 of Ref. [32]. Second, RIS in-plane dispersion is very similar to that of surfons (see Figs. 7 and 9 in Ref. 21]): their energy gap $\Delta_{2} \approx 1.6 K$, and at in-plane momentum $k_{\|}<1.5 \AA^{-1}$ they have almost a quadratic dispersion in Eq. (2) with effective mass $M^{*}$ close to the atomic He mass $M_{4}^{0}=6.7 \cdot 10^{-24} \mathrm{~g}$. Third, similar to surfons, these surface excitations are interpreted as the $\mathrm{He}$ vapor atoms with wave function having large peak just above liquid surface [21], forming a bound state at the surface at zero temperature. At finite temperature these atoms in the bound surface states are quasi-stationary, i.e. they have small finite probability to become delocalized, similar to surfon evaporation at finite temperature studied in Ref. [32]. Thus we suggest that the resonance interface states, obtained numerically in Ref. [21], and the surfons, proposed in Refs. [5, [6], describe the same type of surface excitations with two different approximate approaches. 35]

Therefore, the experimental investigation of the dispersion law of surface excitations, provided by inelastic neutron scattering on He films, is very helpful for detecting surfons and studing their properties. These properties may somewhat differ from those predicted by the semiphenomenological approach of Refs. [5, 6] or approximate numerical calculations of Ref. [21]. The numerical calculations of the dynamics of one He atom approaching the surface and interacting with nearest atoms from the liquid could additionally prove the existence of surfons and even estimate their lifetime. Taking into account the important role of surfon excitations in the physical properties of the surface of liquid helium and, possibly, of other cryogenic liquids, further numerical calculations on this problem are highly need.

The observed additional branch of intensity maxima, giving the in-plane dispersion of surface excitations and approximately coinciding with the dashed line in Figs. [1]3 of possible surfon spectrum, give a strong support of the existence of surfons and suggest their dispersion law. Alternatively, this additional branch could be due to the "breathing mode", obtained in Refs. [16 18, 22] for thin He films. This breathing mode has a different in-plane dispersion, closer to linear rather than quadratic as for surfon. In addition, for thick films there should be several such modes, corresponding to different quantum numbers of dimensional quantization along the z-axis. The monitoring of the evolution of the activation energy of this mode with the change of He film thickness could elucidate the nature of this excitation and completely rule out (or confirm) its breathing-mode origin, but this, probably, requires experimental data with higher energy resolution.

To summarize, we analyze the experimental data on inelastic neutron scattering by thin $\sim 5$-atomic-layer film of liquid helium at three different temperatures. The scattering intensity plot, shown in Figs. [1], suggests a new type of gapped surface excitations with activation energy $\sim 4.5 \mathrm{~K}$ and dispersion similar to that expected for surfons, proposed and investigated semi-phenomenologically in Refs. [5 [8]. Surface excitations with very similar structure and properties were also obtained by numerical calculations and called resonance interface states. 21] 
Before there were only indirect experimental substantiations of surfons, based on temperature dependence of surface tension coefficient [5, 6] and on interaction of surfons with surface electrons [7, 8]. The shown data on inelastic neutron scattering, probably, provide the first direct observation of surfons. However, further experimental and theoretical study is need for the undoubted confirmation of surfons as surface excitations and for the quantitative analysis of their properties.

The authors thank H. Godfrin for providing the partially unpublished experimental data [1] and E. Krotscheck for useful discussions. The work was supported by the program 0033-2018-0001 "Condensed Matter Physics" by the FASO of Russia. A.D.G. thanks RFBR grant \#16-02-00522.
[1] H.J. Lauter and H. Godfrin, private communication.

[2] H. J. Lauter, H. Godfrin, V. L. P. Frank, and P. Leiderer, Phys. Rev. Lett. 68, 2484 (1992).

[3] H. J. Lauter, H. Godfrin, and P. Leiderer, J. Low Temp. Phys. 87, 425 (1992).

[4] B. E. Clements, H. Godfrin, E. Krotscheck, H. J. Lauter, P. Leiderer, V. Passiouk, and C. J. Tymczak, Phys. Rev. B 53, 12242 (1996).

[5] A.M. Dyugaev, P.D. Grigoriev, JETP Lett. 78, 466 (2003) [Pisma v ZhETF 78, 935 (2003)].

[6] A.D. Grigoriev, P.D. Grigoriev, A.M. Dyugaev, J. Low Temp. Phys. 163, 131 (2011); arXiv:0905.2306

[7] P.D. Grigoriev, A.M. Dyugaev, E.V. Lebedeva, JETP 106(2), 316 (2008).

[8] P. D. Grigor'ev, A. M. Dyugaev and E. V. Lebedeva, JETP Letters 87, 106 (2008) [Pisma v ZhETF 87, 114 (2008)].

[9] J.S.Rowlinson and B. Widom, Molecular Theory of cappilarity, Dover Publications, Mineola NY, 2002.

[10] D.O. Edwards and W.F. Saam, Chapter 4 in The free surface of liquid helium, Ed. by D.F. Brewer, Progress in Low Temperature Physics (series), North-Holland Publishing Company, 1978

[11] V.S. Edel'man, Sov. Phys. - Uspehi 130, 676 (1980).

[12] V.B. Shikin and Yu.P. Monarkha, Two-Dimensional Charged Systems in Helium (in Russian), Nauka, Moscow (1989).

[13] Y. Monarkha, K. Kono, Two-Dimensional Coulomb Liquids and Solids, Springer Verlag, 2004.

[14] E. Feenberg, Theory of Quantum Fluids (Academic, New York, 1969).

[15] G. Mahan, Many-Particle Physics, 2nd ed. (Plenum Press, New York, 1990), Ch. 10.

[16] E. Krotscheck, Phys. Rev. B 31, 4258 (1985).

[17] E. Krotscheck, Phys. Rev. B 32, 5713 (1985).

[18] E. Krotscheck and C. J. Tymczak, Phys. Rev. B 45, 217 (1992).

[19] B. E. Clements, E. Krotscheck, and C. J. Tymczak, Phys. Rev. B 53, 12253 (1996).
[20] K.A. Gernoth, J.W. Clark, G. Senger and M.L. Ristig, Phys. Rev. B 49, 15836 (1994).

[21] K. A. Gernoth and M. L. Ristig, Phys. Rev. B 45, 2969 (1992).

[22] B. E. Clements, E. Krotscheck, C. J. Tymczak, J. Low Temp. Phys. 107, 387 (1997).

[23] H.-J. Lauter, in Excitations in Two-Dimensional and Three Dimensional -Quantum Fluids, NATO ASI series, edited by A. F. G. Wyatt aud H. J. Lauter (Plenum, New York, 1991).

[24] L. Pricaupenko and J. Treiner, J. Low Temp.Phys. 101, 809 (1995).

[25] C. E. Campbell, B. E. Clements, E. Krotscheck, and M. Saarela, Phys. Rev. B 55, 3769 (1997).

[26] L. D. Landau and E. M. Lifshitz, Course of Theoretical Physics, Vol. 6: Fluid Mechanics, 2nd ed. ButterworthHeinemann, 1987.

[27] A.F. Andreev, JETP 23, 939 (1966) [Zh. Exp. Teor. Fiz. 50, 1415 (1966)].

[28] C.G. Paine and G.M. Seidel, Phys. Rev. B 46, 1043 (1992).

[29] K.Shirahama, S. Ito, H.Suto and K. Kono, J. Low Temp. Phys. 101, 439 (1995).

[30] M. Saitoh, J. Phys. Soc. Japan 42, 201 (1977).

[31] Russell J. Donnelly and Carlo F. Barenghi, The Observed Properties of Liquid Helium at the Saturated Vapor Pressure, Journal of Physical and Chemical Reference Data 27, 1217 (1998).

[32] A.D. Grigoriev, P.D. Grigoriev, A.M. Dyugaev, A.F. Krutov, Low Temp. Phys. 38, 1005 (2012).

[33] E. Krotscheck, private communication.

[34] M. A. H. Tucker and A. F. G. Wyatt, Journal of Low Temp. Phys. (Springer) 100, 105 (1995).

[35] The second surface excitation mode, proposed in Ref. 20] for deep He, probably, corresponds to the superposition of surfon and surface phonon mode, as it has two maxima of the square of $\mathrm{He}$ wave function, one above and one below the He surface. 